\title{
A novel resveratrol analog PA19 attenuates obesity-induced cardiac and renal injury by inhibiting inflammation and inflammatory cell infiltration
}

\author{
WENXIN ZHANG ${ }^{1}$, HONGJIN CHEN $^{1}$, CHUCHU SUN $^{1}$, BEIBEI WU ${ }^{2}$, BIN BAI $^{1}$, \\ HUI LIU ${ }^{1}$, XIAOOU SHAN ${ }^{2}$, GUANG LIANG ${ }^{1}$ and YALI ZHANG ${ }^{1}$ \\ ${ }^{1}$ Chemical Biology Research Center, School of Pharmaceutical Sciences, Wenzhou Medical University, \\ Wenzhou, Zhejiang $325035 ;{ }^{2}$ The Second Affiliated Hospital and Yuying Children's Hospital of \\ Wenzhou Medical University, Wenzhou, Zhejiang 325000, P.R. China
}

Received June 13, 2018; Accepted March 14, 2019

DOI: $10.3892 / \mathrm{mmr} .2019 .10157$

\begin{abstract}
Obesity is a major global health concern and induces numerous complications, such as heart and kidney injury. Inflammation is an important pathogenic mechanism underlying obesity-associated tissue injury. (1E,4E)-1-\{2,4-Dimethoxy-6-[(E)-4-methoxystyryl]phenyl\}-5(2,4-dimethoxyphenyl)penta-1,4-dien-3-one (PA19) is a novel anti-inflammatory compound synthesized by our research group. In the present study, the efficacy of PA19 in attenuating high-fat diet (HFD)-induced heart and kidney injury was investigated. Heart and kidney pathological injury and fibrosis were detected by hematoxylin and eosin and Sirius red staining, respectively. The expression levels of inflammatory genes and fibrosis-associated protein were determined by reverse transcription-quantitative polymerase chain reaction and western blotting. ELISA was used to detect the level of inflammatory cytokines. Following 20 weeks of HFD treatment, mice exhibited increased lipid accumulation in the serum, heart and kidney injury and fibrosis, and inflammation and inflammatory cell infiltration compared with mice fed a control diet. Conversely, treatment with PA19 during the final 12 weeks of the study significantly reduced the degree of heart and kidney fibrosis and inflammation induced by HFD. The results suggested that PA19 attenuates heart and kidney inflammation and injury induced by HFD, and indicated that PA19 may be a novel therapeutic agent in the treatment of obesity, and obesity-induced cardiac and renal injury.
\end{abstract}

Correspondence to: Professor Yali Zhang, Chemical Biology Research Center, School of Pharmaceutical Sciences, Wenzhou Medical University, Chashan University Town, Central North Road, Wenzhou, Zhejiang 325035, P.R. China

E-mail: ya-li000@163.com

Key words: obesity, inflammation, fibrosis, heart, kidney

\section{Introduction}

Obesity is a chronic disease induced by nutritional and metabolic disorders, and is a global health problem; $5-10 \%$ of cases are due to genetic factors, whereas $90-95 \%$ of cases arise from excessive diets and a lack of exercise (1). Obesity involves the accumulation of adipose tissue in the body and disruption of normal metabolic activities, resulting in tissue damage (2). Obesity leads to the development of a variety of conditions and disorders, including hypertension, insulin resistance, diabetes, inflammation, and obesity-induced heart and kidney injury, thereby markedly impairing human health (3-7).

An increasing number of clinical and animal studies have demonstrated the involvement of the innate immune system and inflammatory responses in the development of obesity-induced heart and kidney injury (8). Previous studies have characterized obesity-associated complications as low-grade chronic inflammatory diseases $(9,10)$. Numerous anti-inflammatory compounds have been used in the treatment of obesity-induced tissue injury, including curcumin, berberine and resveratrol, indicating the importance of inhibiting inflammation in treating obesity-associated complications (11-13).

(1E,4E)-1-\{2,4-Dimethoxy-6-[(E)-4-methoxystyryl]phenyl\}5-(2,4-dimethoxyphenyl)penta-1,4-dien-3-one (PA19) is an analog of resveratrol that was synthesized by the present research group (Fig. 1) (14). It was reported to exhibit more potent effects than resveratrol in suppressing lipopolysaccharide (LPS)-induced tumor necrosis factor- $\alpha$ (TNF- $\alpha$ ) and interleukin (IL)-6 production in mouse peritoneal macrophages $(15,16)$. In the present study, the protective effects of PA19 on obesity-induced heart and kidney injury, and inflammation were investigated in a mouse model of obesity in vivo.

\section{Materials and methods}

Chemicals. PA19 was prepared at a purity of $99.2 \%$ as previously described (12), and dissolved in $0.5 \%$ sodium carboxylmethylcellulose (CMC-Na; Sinopharm Chemical Reagent Co., Ltd., Shanghai, China) for in vivo experiments. 
Animals. A total of 21 male C57BL/6 mice (8-10 weeks old, 18-22 g) and a total of 20 male 18-20 g ICR mice (8-10 weeks old) were obtained from the Animal Center of Wenzhou Medical University (Wenzhou, China). All animal care and experimental procedures complied with the Ordinance in Experimental Animal Management (order no. 1998-02; Ministry of Science and Technology, Beijing, China) and were approved by the Wenzhou Medical University Animal Policy and Welfare Committee (Wenzhou, China). Mice were housed at $22^{\circ} \mathrm{C}$, with $55 \pm 15 \%$ humidity and maintained under a 12 -h light/dark cycle in an SPF environment. All animals were provided with free access to water. Following an acclimatization period of 1 week, mice were randomly assigned into three weight-matched groups for 20 weeks ( $\mathrm{n}=7 /$ group): Mice were fed a standard rodent diet (CON) or a high-fat diet (HFD), or fed a HFD and received intragastric administration of PA19 $(10 \mathrm{mg} / \mathrm{kg} / \mathrm{day})$ via oral gavage on alternate days for the final 12 weeks of the study (HFD + PA19). The mice in the CON and HFD groups received vehicle treatment ( $0.5 \% \mathrm{CMC}-\mathrm{Na}$ solution) as a control. The body weights of the mice were monitored weekly during the 20-week period of the study. The mice were subsequently sacrificed under anesthesia, heart and kidney tissues were fixed in $4 \%$ paraformaldehyde at room temperature for $48 \mathrm{~h}$ for histological, and gene and protein expression analyses.

Reverse transcription-quantitative polymerase chain reaction (RT-qPCR). Total RNA was isolated from tissues using TRIzol $^{\circledR}$ (Invitrogen; Thermo Fisher Scientific, Inc., Waltham, MA, USA); the concentration of RNA was determined using a SpectraMax M5 microplate reader (Molecular Devices, LLC, Sunnyvale, CA, USA) and the purity of the samples was estimated from the optical density ratio (A260/A280, 1.8-2.2). RT was performed using an M-MLV Platinum RT-qPCR kit (Invitrogen; Thermo Fisher Scientific, Inc.) with the reaction parameters of incubation at $65^{\circ} \mathrm{C}$ for $5 \mathrm{~min}, 37^{\circ} \mathrm{C}$ for $52 \mathrm{~min}$ and $70^{\circ} \mathrm{C}$ for $15 \mathrm{~min}$. qPCR was conducted with a total reaction volume of $20 \mu \mathrm{l}$ using $\mathrm{iQ}^{\mathrm{TM}} \mathrm{SYBR}^{\circledR}-$ Green Supermix (Bio-Rad Laboratories, Inc., Hercules, CA, USA) and a MasterCycler Realplex 4 (Eppendorf, Hamburg, Germany). A total of 3 parallel experiments of one sample were conducted. A total of 60 cycles of qPCR were performed. The thermocycling conditions were as follows: Denaturation at $95^{\circ} \mathrm{C}$ for $2 \mathrm{~min}$; annealing at $95^{\circ} \mathrm{C}$ for $15 \mathrm{sec}$ and extension at $60^{\circ} \mathrm{C}$ for $30 \mathrm{sec}$. Primers were obtained from Invitrogen (Thermo Fisher Scientific, Inc.); the sequences are presented in Table I. The relative expression of each gene normalized to $\beta$-actin was determined using the $2^{-\Delta \Delta \mathrm{Cq}}$ method (17). mRNA expression levels were calculated as percentages of the expression in the median tissue from the HFD group.

Western blotting. Heart and kidney tissues from each mouse were homogenized in radioimmunoprecipitation assay (RIPA) buffer (P0013B; Beyotime Institute of Biotechnology, Haimen, China;) with protease inhibitor. The Bradford assay (Bio-Rad Laboratories, Inc.) was performed to determine the concentration of protein. Following boiling in loading buffer for $10 \mathrm{~min}$, total protein samples $(60 \mu \mathrm{g})$ were separated via $10 \%$ SDS-PAGE and transferred onto polyvinylidene difluoride membranes (Bio-Rad Laboratories, Inc.). Membranes were blocked with 5\% milk for $1.5 \mathrm{~h}$ at room temperature and then incubated with primary

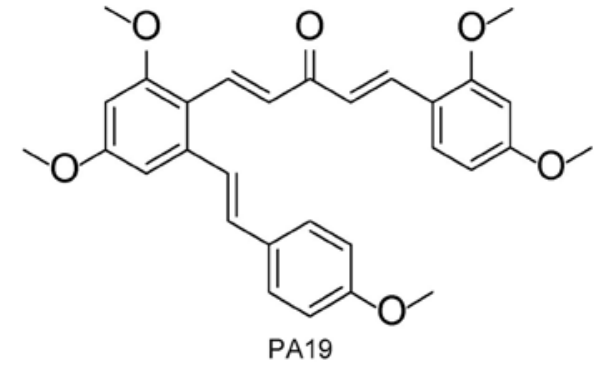

Figure 1.Chemical structure of PA19.PA19,(1E,4E)-1-\{2,4-dimethoxy-6-[(E)4-methoxystyryl]phenyl\}-5-(2,4-dimethoxyphenyl)penta-1,4-dien-3-one.

antibodies overnight at $4^{\circ} \mathrm{C}$. The following primary antibodies were used: Vascular cell adhesion molecule 1 (VCAM-1; 1:1,000; ab134047; Abcam, Cambridge, UK), intercellular adhesion molecule 1 (ICAM-1; 1:1,000; ab171123, Abcam), monocyte chemoattractant protein 1 (MCP-1; 1:1,000; sc-52701, Santa Cruz Biotechnology, Inc., Dallas, TX, USA), collagen 1 (Col-1; 1:1,000; ab34710, Abcam) and $\beta$-actin (1:1,000; ab8226, Abcam). The membranes were subsequently washed in TBST (TBS solution containing $0.2 \%$ Tween-20) and incubated with horseradish peroxidase (HRP)-conjugated secondary antibodies (1:3,000; 7074; Cell Signaling Technology, Inc., Danvers, MA, USA) for $1 \mathrm{~h}$ at room temperature. Blots were visualized using an enhanced chemiluminescence reagent (Bio-Rad Laboratories, Inc.) and protein expression was quantified using Image J software (version 1.42q; National Institutes of Health, Bethesda, MD, USA).

ELISA. Heart and kidney tissues from each mouse were homogenized in RIPA buffer with protease inhibitor, and the Bradford assay was performed to determine the concentration of protein. Following adjustment to equal concentrations, ELISAs were performed on the samples using mouse IL-6 (85-88-7064-76; eBioscience; Thermo Fisher Scientific, Inc.) and mouse IL-1 $\beta$ ELISA kits (70-EK201B/3-96; MultiSciences, Hangzhou, China) according to the manufacturer's protocols.

Heart and kidney function tests. Blood samples were collected through the eyeball after sacrifice of the animals and centrifuged at $3,000 \mathrm{x} \mathrm{g}$ for $15 \mathrm{~min}$ at $4^{\circ} \mathrm{C}$ to isolate the serum. Heart and kidney injury and failure were determined by measuring the serum expression levels of various biochemical markers, including blood urea nitrogen (BUN), serum creatinine $(\mathrm{Cr})$, creatine kinase (CK), CK-muscle/brain (CK-MB), total cholesterol (TCH), alkaline phosphatase (AKP), low density lipoprotein (LDL) and aspartate transaminase (AST) using respective commercial kits (Jiancheng Bioengineering Institute, Nanjing, China).

Heart and kidney histopathology. Kidney and heart tissues were fixed in $4 \%$ paraformaldehyde for $48 \mathrm{~h}$ at room temperature and embedded in paraffin. Samples were sectioned (5- $\mu \mathrm{m}$ thickness) and mounted on slides. Then, sections were stained with H\&E and Sirius Red (Beijing Solarbio Science \& Technology Co., Ltd., Beijing, China) according to the manufacturer's protocols. To analyze the extent of damage, 5 fields of one specimen were observed under a light microscope (magnification, x200; Nikon Corporation, Tokyo, Japan). The area of fibrosis in the heart and 
Table I. Primer sequences for reverse transcription-quantitative polymerase chain reaction.

\begin{tabular}{|c|c|c|c|}
\hline Gene & Species & Forward primer & Reverse primer \\
\hline IL-1 $\beta$ & Mouse & 5'-ACTCCTTAGTCCTCGGCCA-3' & 5'-CCATCAGAGGCAAGGAGGAA-3' \\
\hline IL-6 & Mouse & 5'-GAGGATACCACTCCCAACAGACC-3' & 5'-AAGTGCATCATCGTTGTTCATACA-3' \\
\hline VCAM-1 & Mouse & 5'-TGCCGAGCTAAATTACACATTG-3' & 5'-CCTTGTGGAGGGATGTACAGA-3' \\
\hline ICAM-1 & Mouse & 5'-GCCTTGGTAGAGGTGACTGAG-3' & 5'-GACCGGAGCTGAAAAGTTGTA-3' \\
\hline$\beta$-actin & Mouse & 5'-CCGTGAAAAGATGACCCAGA-3' & 5'-TACGACCAGAGGCATACAG-3' \\
\hline
\end{tabular}

ICAM-1, intercellular adhesion molecule 1; IL, interleukin; VCAM-1, vascular cell adhesion molecule 1.

kidney tissues was determined using Image J software (version 1.42q, National Institutes of Health).

Immunohistochemistry. Heart and kidney tissues were sectioned as aforementioned, deparaffinized in xylene and rehydrated in a graded alcohol series. Antigen retrieval was performed in $10 \mathrm{mM}$ sodium citrate buffer ( $\mathrm{pH}$ 6.5) by microwaving (10 min). Endogenous peroxidase activity was blocked by incubation in $3 \%$ hydrogen peroxide for $30 \mathrm{~min}$ at room temperature. Sections were then blocked for $30 \mathrm{~min}$ at $37^{\circ} \mathrm{C}$ in $1 \%$ bovine serum albumin (Sigma-Aldrich; Merck KGaA, Darmstadt, Germany) dissolved in phosphate buffer solution, prior to incubation with primary anti-epidermal growth factor-like module-containing mucin-like hormone receptor-like 1 (F4/80) antibody (1:200; sc-377009; Santa Cruz Biotechnology, Inc.) at $4^{\circ} \mathrm{C}$ overnight. Then, sections were incubated with HRP-conjugated secondary antibodies (1:200; 33201ES60; Yeasen, Shanghai, China) at $37^{\circ} \mathrm{C}$ for $30 \mathrm{~min}$, prior to immersion in 3'3-diaminobenzidine solution (OriGene Technologies, Inc., Beijing, China) for $1 \mathrm{~min}$. Finally, sections were stained with hematoxylin for $5 \mathrm{~min}$ at room temperature, dehydrated and mounted, and images were captured using a light microscope ( 5 fields of one specimen were randomly selected; magnification, x200, Nikon Corporation).

Acute toxicity assay. The 20 male 18-20 g ICR mice were randomly divided into two experimental groups: CON group and PA19 group. CON and PA group mice were received $0.5 \%$ CMC-Na and $500 \mathrm{mg} / \mathrm{kg} \mathrm{PA}$, respectively. The treatments were administered once by oral gavage. Animals were closely observed every $12 \mathrm{~h}$ following administration on general behavior; mortality and body weights were also measured.

Statistical analysis. All data were obtained from three independent experiments. The results were presented as the mean \pm standard error of the mean. All data were analyzed using GraphPad Pro Prism 5.01 (GraphPad Software, Inc., La Jolla, CA, USA). One-way analysis of variance followed by a Dunnett's post-hoc test was employed to analyze the differences between multiple groups. $\mathrm{P}<0.05$ was considered to indicate a statistically significant difference.

\section{Results}

PA19 reduces the expression of obesity-induced kidney and heart biochemical markers in the serum. Heart and kidney tissues and serum were collected from mice following CON or HFD feeding for 20 weeks. As presented in Table II, animals fed with the HFD exhibited significantly increased serum expression levels of lipid accumulation markers ( $\mathrm{TCH}$ and LDL), renal function markers (BUN and $\mathrm{Cr}$ ) and cardiac function markers (CK, CK-MB, AKP and AST) compared with the CON group; however, treatment with PA19 $(10 \mathrm{mg} / \mathrm{kg})$ suppressed the increased expression of these markers. An acute toxicity assay was conducted to determine the effects of chronic PA19 treatment in vivo; it was demonstrated that intragastric administration of PA19 (500 mg/ kg) did not significantly alter body weight (Fig. S1) or the serum expression levels of the aforementioned biochemical markers (Table SI) compared with treatment with $0.5 \% \mathrm{CMC}-\mathrm{Na}$. The results indicated that PA19 treatment at the selected dose was safe for mice and attenuated the increased serum expression levels of functional biomarkers following exposure to an HFD.

PA19 reduces obesity-induced heart and kidney injury and fibrosis. The cardioprotective effects of PA19 were investigated by determining the effects of PA19 treatment on obesity-induced alterations in the morphology of cardiac tissue. H\&E staining revealed that the heart tissue of mice in the HFD group presented structural abnormalities that were not observed in the PA19-treated group, including broken fibers and irregular cellular structures (Fig. 2A). Additionally, Sirius Red staining revealed that PA19 treatment attenuated HFD-induced collagen deposition in heart tissues and significantly reduced fibrosis compared with the control (Fig. 2B and C). It was demonstrated that feeding with the HFD induced significant increases in the levels of Col-1 mRNA and protein expression in heart tissue compared with the CON group, but were suppressed by PA19 treatment (Fig. 2D, E and S2A).

H\&E staining of kidney tissue revealed that the HFD group exhibited glomerular shrinkage and expansion of the mesangial matrix compared with the CON group (Fig. 3A). Conversely, treatment with PA19 markedly improved obesity-induced renal injury. The extent of fibrosis in the kidneys induced by HFD was also evaluated. Sirius Red staining revealed notable collagen deposition in the kidney tissues of HFD mice (Fig. 3B and C), in addition to significant increases in the expression of Col-1 at the mRNA and protein levels compared with the CON group (Fig. 3D, E and S2B). PA19 treatment significantly reduced the extent of HFD-induced fibrosis and decreased the expression of Col-1 in kidney tissue. The results 
Table II. Blood biochemical data following 20 weeks on the CON, or the HFD with or without PA19 treatment.

\begin{tabular}{lcrr}
\hline Factor & \multicolumn{1}{c}{ CON } & \multicolumn{1}{c}{ HFD } & PA19+HFD \\
\hline TCH (mmol/l) & $97.57 \pm 5.66$ & $254.30 \pm 6.64^{\mathrm{b}}$ & $201.70 \pm 9.95^{\mathrm{c}}$ \\
LDL (mmol/l) & $0.53 \pm 0.06$ & $1.40 \pm 0.07^{\mathrm{b}}$ & $0.98 \pm 0.07^{\mathrm{c}}$ \\
BUN (mmol/l) & $8.16 \pm 1.01$ & $18.61 \pm 0.80^{\mathrm{b}}$ & $14.91 \pm 0.48^{\mathrm{c}}$ \\
Cr (mmol/l) & $9.27 \pm 1.82$ & $57.20 \pm 5.79^{\mathrm{b}}$ & $36.21 \pm 5.22^{\mathrm{c}}$ \\
CK-MB (U/l) & $748.6 \pm 146.4$ & $1,324.0 \pm 56.51^{\mathrm{a}}$ & $1,288.0 \pm 54.60$ \\
CK (U/l) & $0.17 \pm 0.11$ & $0.75 \pm 0.08^{\mathrm{b}}$ & $0.47 \pm 0.07^{\mathrm{c}}$ \\
AKP (U/l) & $0.033 \pm 0.01$ & $0.550 \pm 0.03^{\mathrm{b}}$ & $0.440 \pm 0.02$ \\
AST (U/l) & $0.044 \pm 0.01$ & $0.087 \pm 0.01^{\mathrm{a}}$ & $0.049 \pm 0.01^{\mathrm{c}}$
\end{tabular}

${ }^{\mathrm{a}} \mathrm{P}<0.05 \mathrm{vs}$. $\mathrm{CON} ;{ }^{\mathrm{b}} \mathrm{P}<0.01 \mathrm{vs}$. $\mathrm{CON}$; ${ }^{\mathrm{c}} \mathrm{P}<0.05 \mathrm{vs}$. HFD. Data are presented as the mean \pm standard error of the mean. AKP, alkaline phosphatase; AST, aspartase transaminase; BUN, blood urea nitrogen; CK-MB, creatine kinase-muscle/brain; CON, control diet; Cr, serum creatinine; HFD, high-fat diet; LDL, low density lipoprotein; PA19, (1E,4E)-1-\{2,4-dimethoxy-6-[(E)-4-methoxystyryl]phenyl\}-5-(2,4-dimethoxyphenyl) penta-1,4-dien-3-one; TCH, total cholesterol.

A

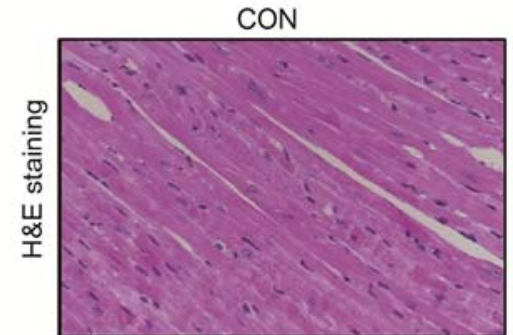

B

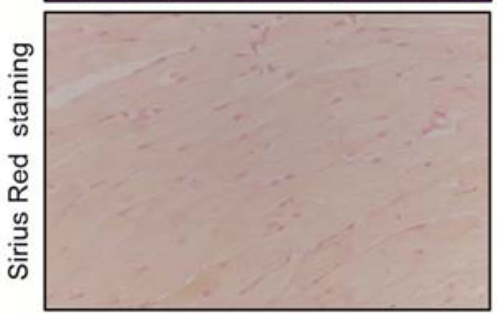

C

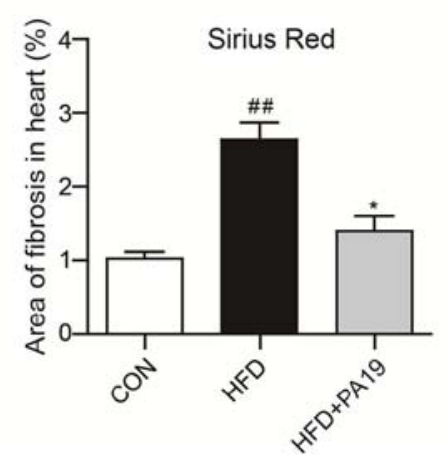

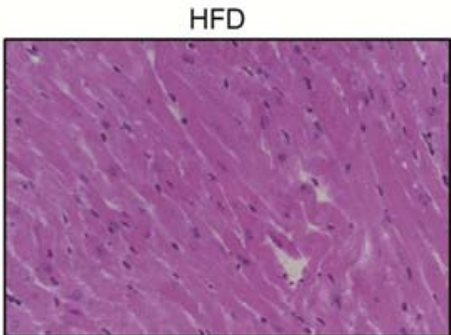

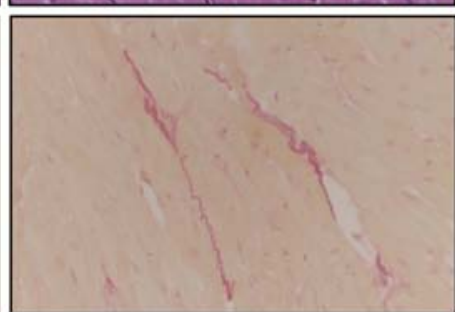

$\mathrm{D}$

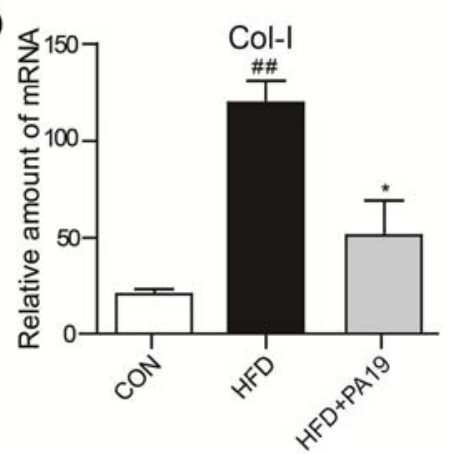

HFD+PA19
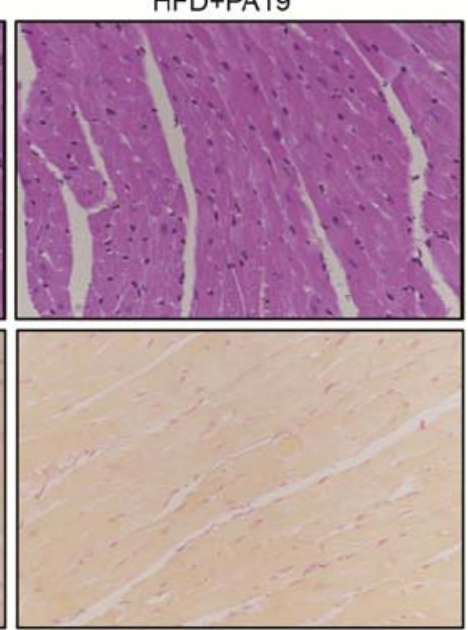

E

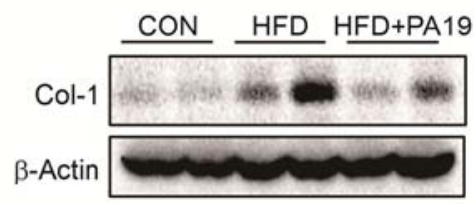

Figure 2. PA19 reduces HFD-induced cardiac injury and fibrosis. Obesity was induced over 20 weeks in male C57BL/6 mice with an HFD, plus oral gavage of PA19 (10 mg/kg) or vehicle ( $0.5 \%$ sodium carboxymethylcellulose) once every 2 days during the final 12 weeks. Representative images of (A) H\&E and (B) Sirius Red staining of heart tissues (magnification, x200). (C) Relative area of fibrosis in cardiac tissues. (D) mRNA expression of Col-1 in heart tissues as determined by reverse transcription-quantitative polymerase chain reaction. (E) Protein expression of Col-1 in cardiac tissues as determined by western blotting. $\mathrm{N}=7$ /group. Data are presented as the mean \pm standard error of the mean. ${ }^{*} \mathrm{P}<0.05$ vs. HFD group; ${ }^{\# \#} \mathrm{P}<0.01$ vs. CON group. Col-1, collagen 1 ; CON, control diet; HFD, high-fat diet; PA19, (1E,4E)-1-\{2,4-dimethoxy-6-[(E)-4-methoxystyryl]phenyl\}-5-(2,4-dimethoxyphenyl)penta-1,4-dien-3-one.

suggested that treatment with PA19 protects heart and kidney tissue from obesity-induced injury and fibrosis.

PA19 reduces obesity-induced myocardial and renal inflammation. The association between the potential cardio- and renoprotective effects of PA19 against obesity-induced damage and its anti-inflammatory properties was investigated. Immunohistochemical staining of F4/80 expression revealed that there was marked HFD-induced inflammatory cell infiltration of cardiac tissue; however, the percentage of $\mathrm{F} 4 / 80^{+}$cells in 
A

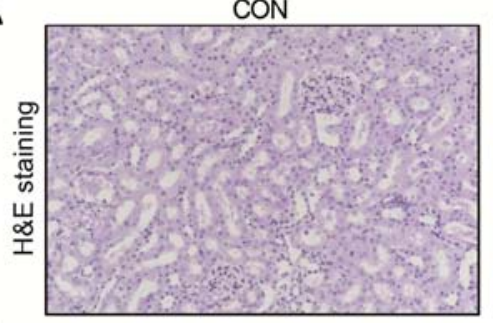

B

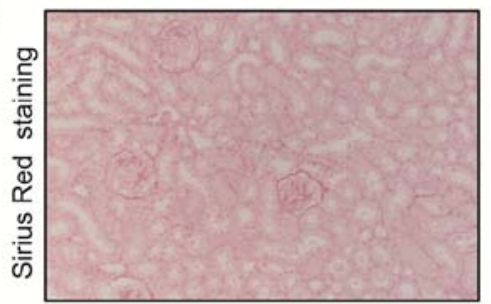

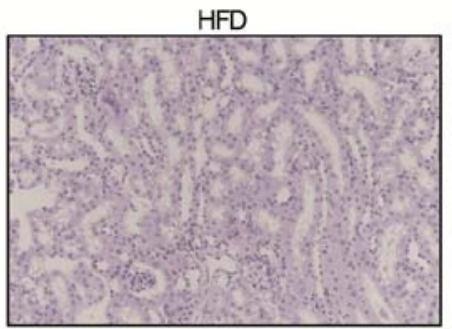
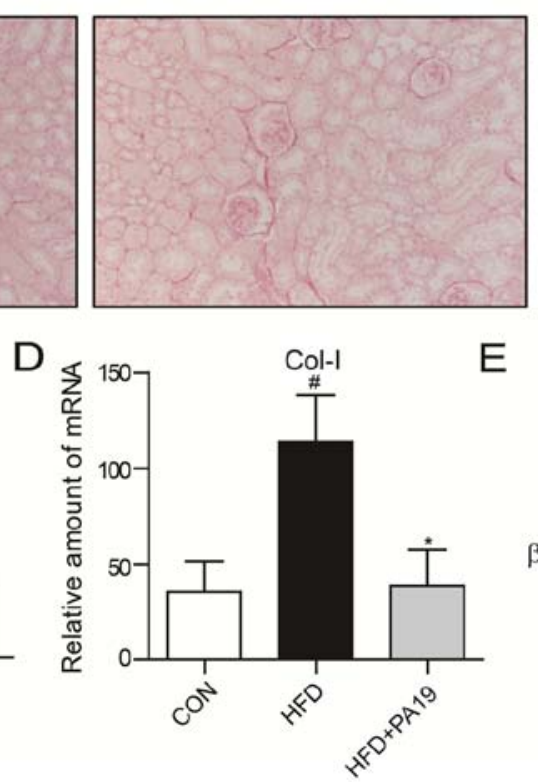

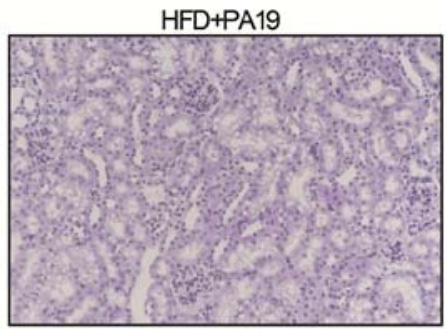

E

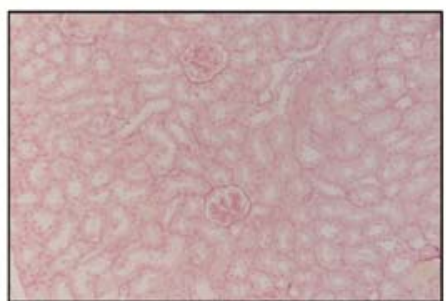

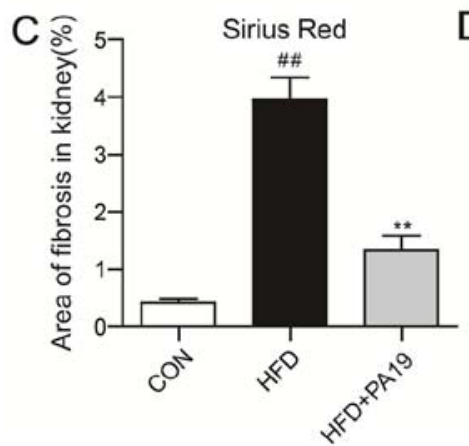

Figure 3. PA19 reduces HFD-induced renal injury and fibrosis. Representative images of (A) H\&E and (B) Sirius Red staining of kidney tissues (magnification, $\mathrm{x} 200$ ). (C) Relative area of fibrosis in renal tissues. (D) mRNA expression of Col-1 in kidney tissues as determined by reverse transcription-quantitative polymerase chain reaction. (E) Protein expression of Col-1 in renal tissues as determined by western blotting. N=7/group. Data are presented as the mean \pm standard error of the mean. ${ }^{*} \mathrm{P}<0.05,{ }^{* *} \mathrm{P}<0.01$ vs. HFD group; ${ }^{*} \mathrm{P}<0.05,{ }^{\# \#} \mathrm{P}<0.05$ vs. CON group. Col-1, collagen 1 ; CON, control diet; HFD, high-fat diet; PA19, (1E,4E)-1-\{2,4-dimethoxy-6-[(E)-4-methoxystyryl]phenyl\}-5-(2,4-dimethoxyphenyl)penta-1,4-dien-3-one.

tissue samples from the HFD + PA19 group was significantly lower compared with in the HFD group (Fig. 4A and B). Additionally, RT-qPCR, ELISA and western blot analyses demonstrated that HFD exposure significantly increased the mRNA and protein expression levels of inflammatory cytokines (IL-6 and IL-1 $\beta$ ), chemokines (MCP-1) and adhesion molecules (VCAM-1 and ICAM-1) in heart tissue compared with in the CON group (Fig. 4C-J). Conversely, PA19 treatment significantly suppressed the HFD-induced upregulation of these markers.

As presented in Fig. 5A and B, feeding with the HFD induced a marked increase in the number of $\mathrm{F} 4 / 80^{+}$inflammatory cells in the renal sections; however, inflammatory cell infiltration was significantly attenuated by treatment with PA19. As presented in Fig. 5C-J, the expression levels of inflammatory cytokines, chemokines and adhesion molecules were significantly increased in kidney tissues from HFD mice compared with the CON group, but were suppressed by PA19 treatment. Of note, the protein expression of IL-1 $\beta$ was not significantly reduced following PA19 administration. Collectively, these results indicated that PA19 exhibits anti-inflammatory properties in cardiac and renal tissues in vivo.

\section{Discussion}

Obesity is a global health issue that may lead to numerous associated disorders, including insulin resistance, diabetes and inflammation (18); therefore, it is important to identify novel approaches to treat obesity-associated complications. In the present study, the protective effects of PA19, a resveratrol analog, were investigated in an established mouse model of obesity. It was revealed that PA19 attenuated obesity-induced lipid accumulation in the serum, injury, collagen deposition, inflammatory cytokine expression and inflammatory cell infiltration in the heart and kidneys. These findings suggested that PA19 exhibits cardio- and renoprotective effects against obesity-induced injury via the inhibition of inflammatory responses.

Long-term over-nutrition commonly leads to obesity and associated complications, such as heart and kidney injury (19). Increased fat accumulation progressively impairs arterial function and thickening of the blood vessel wall reduces blood flow; thus, systemic blood circulation is reduced, and the risk of thrombosis and burden on the heart are increased $(20,21)$. Thickening of the walls of renal blood vessels increases renal blood pressure and burden on the kidney. Consequentially, glomerular hypertension and hyperfiltration can occur, commonly leading to the development of glomerulosclerosis $(22,23)$. The results of the present study revealed that mice fed an HFD exhibited lipid accumulation in the serum, with heart and kidney injury, whereas treatment with PA19 attenuated these effects. 
A

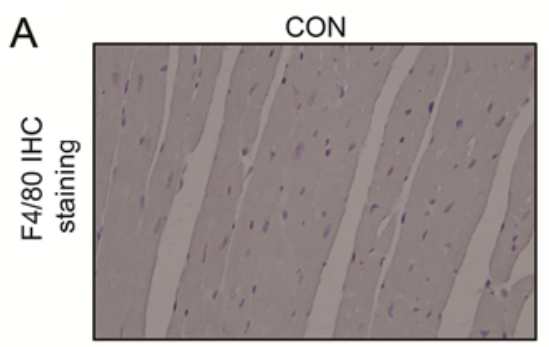

B

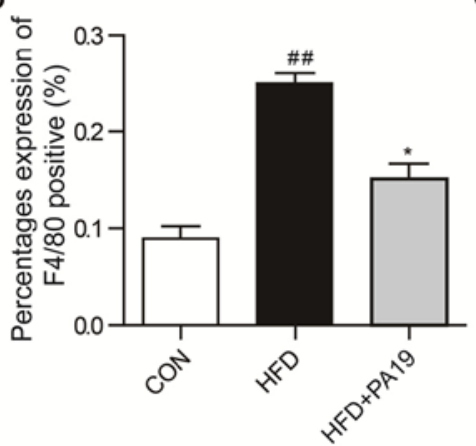

E
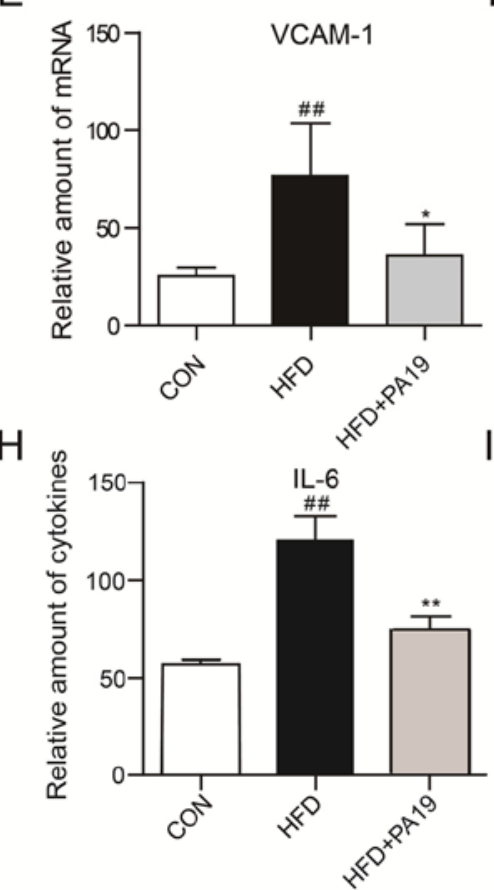

C

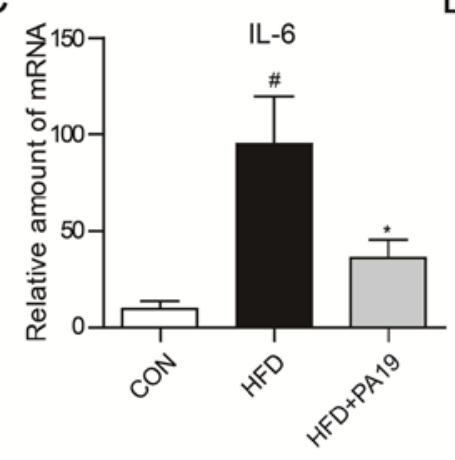

F
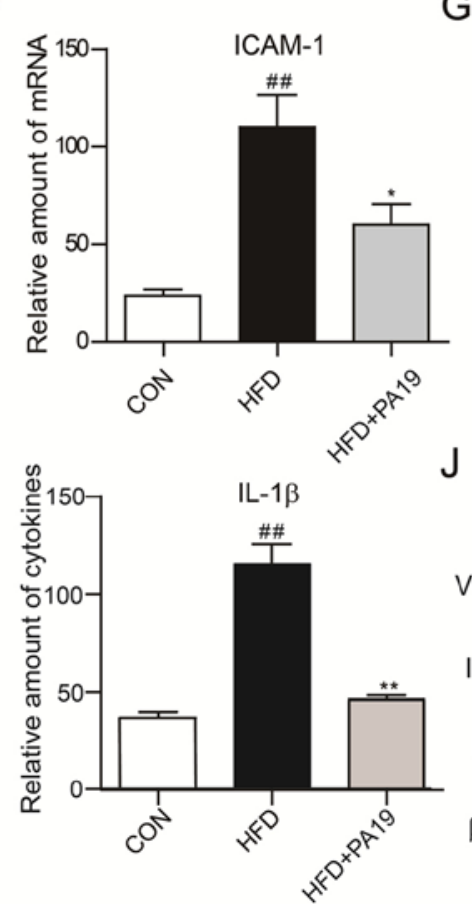

D

G
HFD
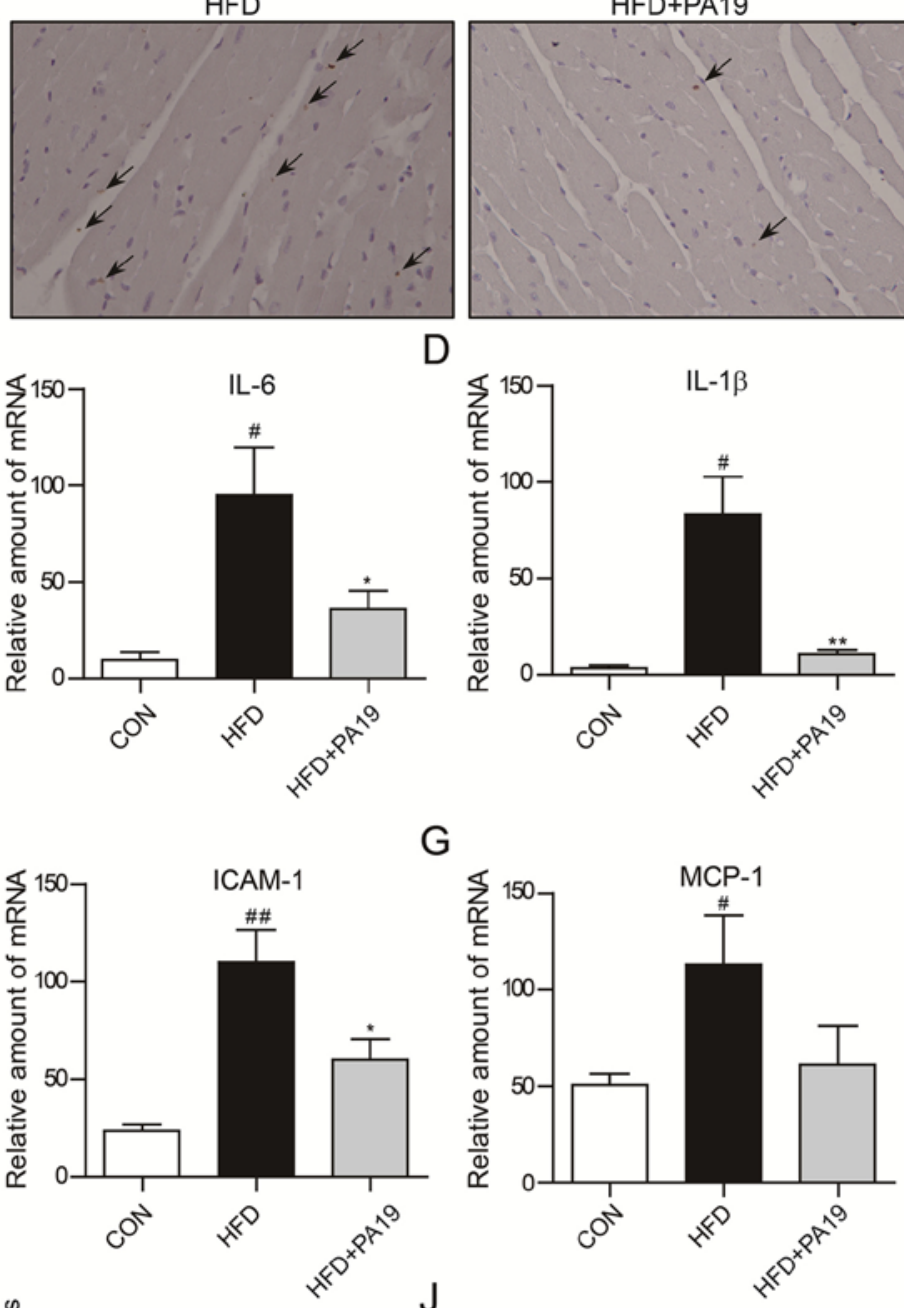

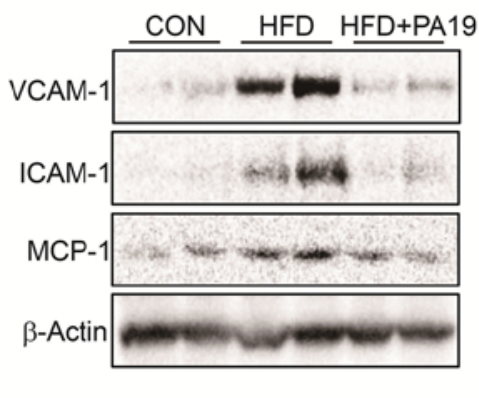

Figure 4. PA19 reduces HFD-induced cardiac inflammation. Representative images of (A) anti-F4/80 staining of heart tissues (magnification, x200). (B) Quantification of F4/80 expression in cardiac tissue sections. mRNA expression of (C) IL-6, (D) IL-1 $\beta$, (E) VCAM-1, (F) ICAM-1 and (G) MCP-1 in heart tissues as determined by reverse transcription-quantitative polymerase chain reaction. Protein expression of (H) IL- 6 and (I) IL-1 $\beta$ as determined by ELISA. (J) Protein expression of VCAM-1, ICAM-1 and MCP-1 in cardiac tissues as determined by western blotting. N=7/group. Data are presented as the mean \pm standard error of the mean. ${ }^{*} \mathrm{P}<0.05,{ }^{* *} \mathrm{P}<0.01$ vs. HFD group; ${ }^{\#} \mathrm{P}<0.05,{ }^{\# \#} \mathrm{P}<0.01$ vs. CON group. CON, control diet; F4/80, epidermal growth factor-like module-containing mucin-like hormone receptor-like 1; HFD, high-fat diet; ICAM-1, intercellular adhesion molecule 1; IL, interleukin; MCP-1, monocyte chemoattractant protein 1; PA19, (1E,4E)-1-\{2,4-dimethoxy-6-[(E)-4-methoxystyryl]phenyl\}-5-(2,4-dimethoxyphenyl)penta-1,4-dien-3-one; VCAM-1, vascular cell adhesion molecule 1.

Fibrosis is an important pathological process in the development of obesity-associated complications. Renal fibrosis is the histological manifestation of a progressive and usually irreversible process resulting in end-stage kidney disease (24). Cardiac fibrosis is associated with metabolic dysfunction, and may contribute to the increased incidence of heart failure associated with obesity $(25,26)$. Col-1 is a major contributor to fibrosis; it has been reported that excessive cardiac Col-1 synthesis and deposition may be involved in the progression of myocardial fibrosis that accompanies the development of heart failure in patients with hypertensive heart disease (27). The expression of Col-1 has been reported to be markedly increased in various types of renal fibrosis $(28,29)$. Sirius Red staining, and RT-qPCR and western blot analyses revealed that PA19 treatment suppressed the collagen deposition and upregulation of Col-1 


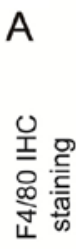

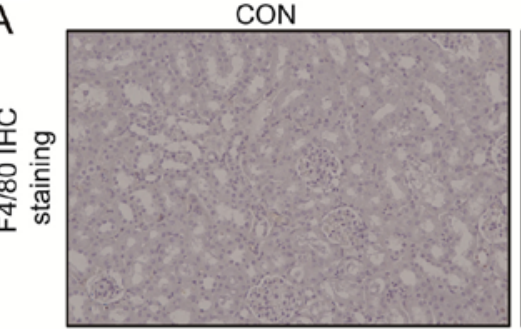

B

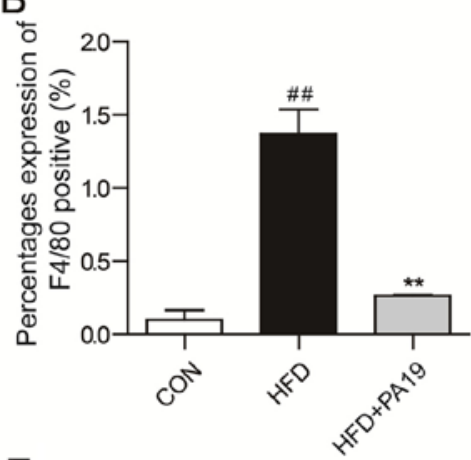

E
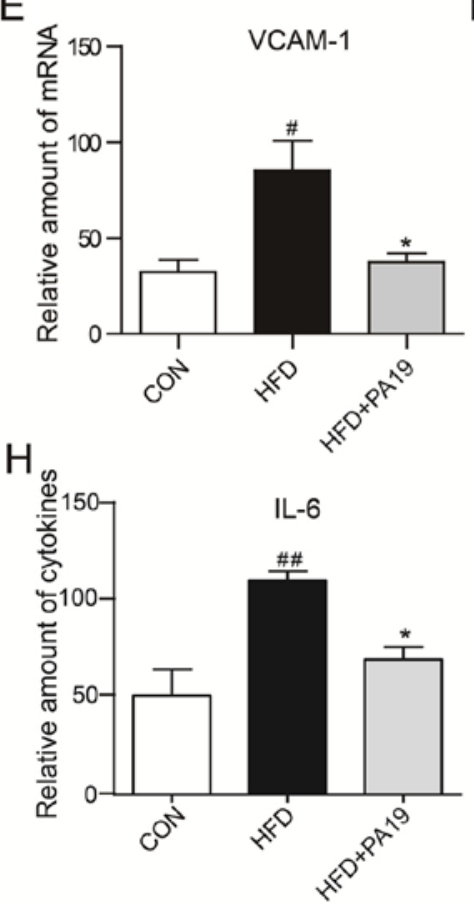

C

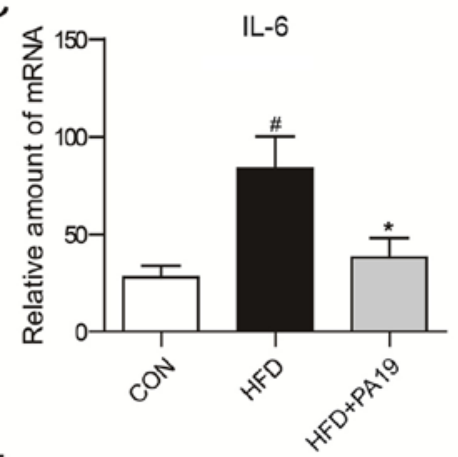

$\mathrm{F}$
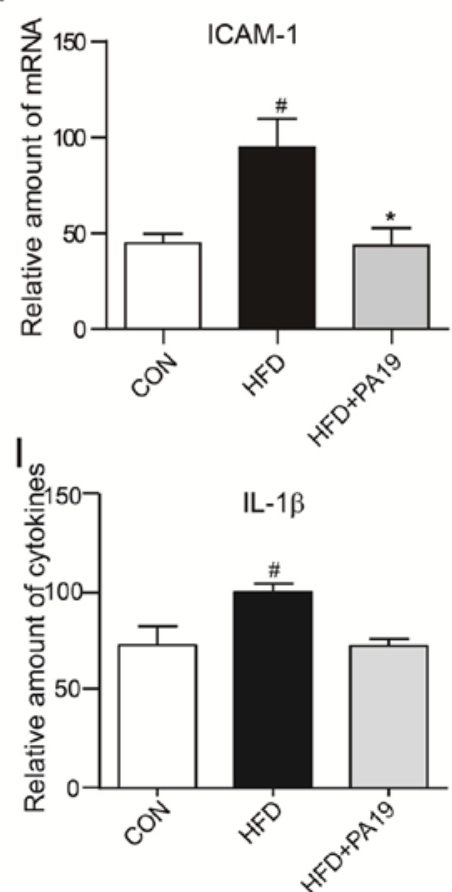

HFD

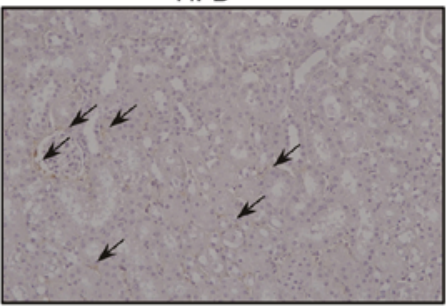

D

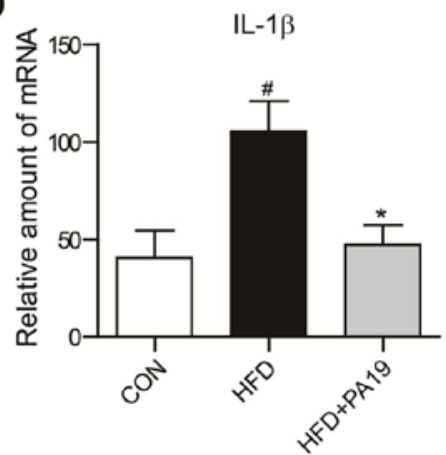

G

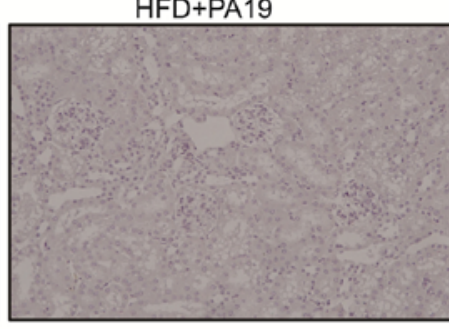

$\S 1507 \quad$ MCP-1

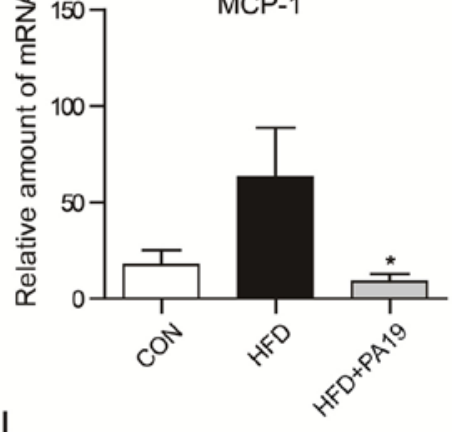

$J$

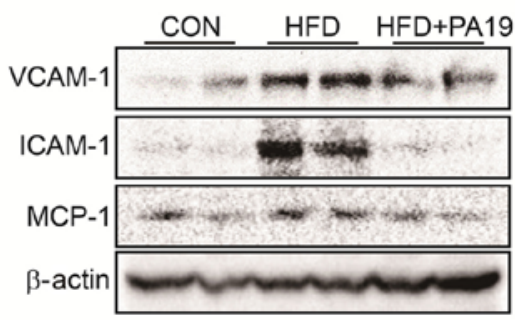

Figure 5. PA19 reduces HFD-induced renal inflammation. Representative images of (A) anti-F4/80 staining of kidney tissues (magnification, x200), (B) Quantification of F4/80 expression in renal tissue sections. mRNA expression of (C) IL-6, (D) IL-1 $\beta$, (E) VCAM-1, (F) ICAM-1 and (G) MCP-1 in kidney tissues as determined by reverse transcription-quantitative polymerase chain reaction. Protein expression of (H) IL-6 and (I) IL-1 $\beta$ as determined by ELISA. (J) Protein expression of VCAM-1, ICAM-1 and MCP-1 in renal tissues as determined by western blotting. N=7/group. Data are presented as the mean \pm standard error of the mean. ${ }^{*} \mathrm{P}<0.05,{ }^{* *} \mathrm{P}<0.01$ vs. HFD group; ${ }^{\#} \mathrm{P}<0.05,{ }^{\#} \mathrm{P}<0.01$ vs. CON group. CON, control diet; F4/80, epidermal growth factor-like module-containing mucin-like hormone receptor-like 1; HFD, high-fat diet; ICAM-1, intercellular adhesion molecule 1; IL, interleukin; MCP-1, monocyte chemoattractant protein 1; PA19, (1E,4E)-1-\{2,4-dimethoxy-6-[(E)-4-methoxystyryl]phenyl\}-5-(2,4-dimethoxyphenyl)penta-1,4-dien-3-one; VCAM-1, vascular cell adhesion molecule 1.

in heart and kidney tissues in HFD mice. These findings suggested that PA19 effectively inhibits obesity-induced tissue injury and fibrosis.

Obesity is characterized as a low-grade chronic inflammatory disease $(9,10)$. This inflammatory state is reflected by the increased production of inflammatory cytokines and the infiltration of inflammatory cells into tissues (9). Cells that infiltrate tissues are typically highly activated, producing proinflammatory cytokines and chemoattractants $(26,30)$, consequentially attracting additional inflammatory cells that secrete more proinflammatory cytokines. In the present study, staining of heart and kidney tissues from HFD mice revealed the infiltration of $\mathrm{F} 4 / 80^{+}$cells, and increased expression levels of inflammatory cytokines, chemokines and adhesion molecules compared with in tissues from CON mice; the effects were attenuated by PA19 treatment. 
Resveratrol is a natural product originating from grapes and other plants (31). Resveratrol has received increasing attention as a potential therapy in the prevention and treatment of atherosclerosis, cardiovascular and cerebrovascular diseases, due to its anti-inflammatory properties and low toxicity (32-34). Furthermore, various studies have reported that resveratrol may attenuate obesity and associated complications via a number of mechanisms $(35,36)$; however, the biological activity of resveratrol is limited by its photosensitivity and metabolic instability (37). In our previous study, a series of resveratrol-curcumin hybrids were synthesized, and their anti-inflammatory properties were evaluated in vitro and in vivo (14). It was demonstrated that one compound, PA19, was more effective than resveratrol in suppressing the LPS-induced production of TNF- $\alpha$ and IL-6 (14). In the present study, it was revealed that PA19 attenuated obesity-induced heart and kidney injury in HFD-fed mice, potentially via the inhibition of inflammation and cellular infiltration.

In conclusion, it was demonstrated that mice fed with an HFD exhibited lipid accumulation, and injury, fibrosis, inflammation and inflammatory cell infiltration in heart and kidney tissues; these effects were attenuated by treatment with PA19. The findings of the present study indicated the contribution of inflammatory processes in the development of obesity-associated cardiac and renal damage, and suggested that the anti-inflammatory compound PA19 may be a potential therapeutic agent in the treatment of obesity-associated complications.

\section{Acknowledgements}

Not applicable.

\section{Funding}

The present study was financially supported by the National Natural Science Funding of China (grant nos. 81770850 to XS, 81872918 to $\mathrm{YZ}$, and 81503123 to YZ) and Zhejiang Provincial Natural Science Foundation of China (grant nos. LY18H310011 to YZ, and LY17H050007 to XS).

\section{Availability of data and materials}

The datasets from current study are available from the corresponding author on reasonable request.

\section{Authors' contributions}

WZ, HC and YZ designed the study. WZ, HC, CS and BW performed the experiments. $\mathrm{BB}$ and HL conducted the statistical analyses. XS and GL participated in the analyzing of results. WZ and $\mathrm{YZ}$ wrote and revised the manuscript. All authors read and approved the final manuscript.

\section{Ethics approval and consent to participate}

All animal care and experimental procedures complied with the Ordinance in Experimental Animal Management and were approved by the Wenzhou Medical University Animal Policy and Welfare Committee.

\section{Patient consent for publication}

Not applicable.

\section{Competing interests}

The authors declare that they have no competing interests.

\section{References}

1. Long JR, Shu XQ, Cai Q, Wen W, Kataoka N, Gao YT and Zheng W: CYP19A1 genetic polymorphisms may be associated with obesity-related phenotypes in Chinese women. Int J Obes (Lond) 31: 418-423, 2007.

2. Polyzos SA, Kountouras J and Mantzoros CS: Adipose tissue, obesity and non-alcoholic fatty liver disease. Minerva Endocrinol 42: 92-108, 2017.

3. Roberts CK, Hevener AL and Barnard RJ: Metabolic syndrome and insulin resistance: Underlying causes and modification by exercise training. Compr Physiol 3: 1-58, 2013.

4. Turer CB, Brady TM and de Ferranti SD: Obesity, hypertension, and dyslipidemia in childhood are key modifiable antecedents of adult cardiovascular disease: A call to action. Circulation 137: 1256-1259, 2018

5. Abderrahmani A, Yengo L, Caiazzo R, Canouil M, Cauchi S, Raverdy V, Plaisance V, Pawlowski V, Lobbens S, Maillet J, et al: Increased hepatic PDGF-AA signaling mediates liver insulin resistance in obesity associated type 2 diabetes. Diabetes 67 : 1310-1321, 2018

6. Kenchaiah S, Evans JC, Levy D, Wilson PW, Benjamin EJ, Larson MG, Kannel WB, and Vasan RS: Obesity and the risk of heart failure. N Engl J Med 347: 305-313, 2002.

7. Wahba IM and Mak RH: Obesity and obesity-initiated metabolic syndrome: Mechanistic links to chronic kidney disease. Clin J Am Soc Nephrol 2: 550-562, 2007.

8. de Heredia FP, Gómez-Martínez S and Marcos A: Obesity, inflammation and the immune system. Proc Nutr Soc 71: 332-338, 2012.

9. Cancello R and Clement K: Is obesity an inflammatory illness? Role of low-grade inflammation and macrophage infiltration in human white adipose tissue. BJOG 113: 1141-1147, 2006.

10. Graziani F, Cialdella P, Liuzzo G, Basile E, Brugaletta S, Pedicino D, Leccesi L, Guidone C, Iaconelli A, Mingrone G, et al: Cardiovascular risk in obesity: Different activation of inflammation and immune system between obese and morbidly obese subjects. Eur J Intern Med 22: 418-423, 2011.

11. Vors C, Couillard C, Paradis M, Gigleux I, Marin J, Vohl M, Couture P and Lamarche B: Supplementation with resveratrol and curcumin does not affect the inflammatory response to a high-fat meal in older adults with abdominal obesity: A randomized, placebo-controlled crossover trial. J Nutr 148: 379-388, 2018.

12. Kim WS, Lee YS, Cha SH, Jeong HW, Choe SS, Lee MR, Oh GT, Park HS, Lee KU, Lane MD and Kim JB: Berberine improves lipid dysregulation in obesity by controlling central and peripheral AMPK activity. Am J Physiol Endocrinol Metab 296: E812-E819, 2009.

13. Rossi EL, Khatib SA, Doerstling SS, Bowers LW, Pruski M, Ford NA, Glickman RD, Niu M, Yang P, Cui Z, et al: Resveratrol inhibits obesity-associated adipose tissue dysfunction and tumor growth in a mouse model of postmenopausal claudin-low breast cancer. Mol Carcinog 57: 393-407, 2018.

14. Pan J, Xu T, Xu F, Zhang Y, Liu Z, Chen W, Fu W, Dai Y, Zhao Y, Feng $\mathbf{J}$ and Liang G: Development of resveratrol-curcumin hybrids as potential therapeutic agents for inflammatory lung diseases. Eur J Med Chem 125: 478-491, 2017.

15. Zong Y, Sun L, Liu B, Deng YS, Zhan D, Chen YL, He Y, Liu J, Zhang ZJ, Sun J and Lu D: Resveratrol inhibits LPS-induced MAPKs activation via activation of the phosphatidylinositol 3-kinase pathway in murine RAW 264.7 macrophage cells. PLoS One 7: e44107, 2012.

16. Lee SM, Zhang Y, Tsuchiya H, Smalling R, Jetten AM and Wang L: Small heterodimer partner/neuronal PAS domain protein 2 axis regulates the oscillation of liver lipid metabolism. Hepatology 61: 497-505, 2015.

17. Livak KJ and Schmittgen TD: Analysis of relative gene expression data using real-time quantitative PCR and the 2(-Delta Delta C(T)) method. Methods 25: 402-408, 2001. 
18. Latifi SM, Karandish M, Shahbazian H, Taha JM, Cheraghian B and Moradi M: Prevalence of Metabolically Healthy Obesity (MHO) and its relation with incidence of metabolic syndrome, hypertension and type 2 diabetes amongst individuals aged over 20 years in Ahvaz: A 5 year cohort study (2009-2014). Diabetes Metab Syndr 11 (Suppl 2): S1037-S1040, 2017.

19. Siddeek B, Li N, Mauduit C, Chehade H, Rigal E, Tolsa J, Armengaud J, Yzydorczyk C, Benahmed M, Vergely C and Simeoni U: Transient postnatal over nutrition induces long-term alterations in cardiac NLRP3-inflammasome pathway. Nutr Metab Cardiovasc Dis 28: 944-951, 2018.

20. Blokhin IO and Lentz SR: Mechanisms of thrombosis in obesity. Curr Opin Hematol 20: 437-444, 2013.

21. Köchli S, Endes K, Infanger D, Zahner L and Hanssen H: Obesity, blood pressure, and retinal vessels: A meta-analysis. Pediatrics 141: e20174090, 2018.

22. Davis S, Nehus E, Inge T, Zhang W, Setchell K and Mitsnefes M: Effect of bariatric surgery on urinary sphingolipids in adolescents with severe obesity. Surg Obes Relat Dis 14: 446-451, 2018

23. Hall JE, Henegar JR, Dwyer TM, Liu J, Da Silva AA, Kuo J and Tallam L: Is obesity a major cause of chronic kidney disease? Adv Ren Replace Ther 11: 41-54, 2004.

24. Sowers JR: Metabolic risk factors and renal disease. Kidney Int 71: 719-720, 2007.

25. Cavalera $\mathbf{M}$, Wang $\mathbf{J}$ and Frangogiannis NG: Obesity, metabolic dysfunction, and cardiac fibrosis: Pathophysiological pathways, molecular mechanisms, and therapeutic opportunities. Trans Res 164: 323-335, 2014

26. Fang Q, Wang J, Zhang Y, Wang L, Li W, Han J, Huang W, Liang $G$ and Wang Y: Inhibition of myeloid differentiation factor-2 attenuates obesity-induced cardiomyopathy and fibrosis. Biochim Biophys Acta Mol Basis Dis 1864: 252-262, 2018.

27. Querejeta R, López B, González A, Sánchez E, Larman M, Martinez Ubago JL and Díez J: Increased collagen type I synthesis in patients with heart failure of hypertensive origin: Relation to myocardial fibrosis. Circulation 110: 1263-1268, 2004.

28. Ohashi K, Iwatani H, Kihara S, Nakagawa Y, Komura N, Fujita K, Maeda N, Nishida M, Katsube F, Shimomura I, et al: Exacerbation of albuminuria and renal fibrosis in subtotal renal ablation model of adiponectin-knockout mice. Arterioscler Thromb Vasc Biol 27: 1910-1917, 2007.
29. Stokes MB, Holler S, Cui Y, Hudkins KL, Eitner F, Fogo A and Alpers CE: Expression of decorin, biglycan, and collagen type I in human renal fibrosing disease. Kidney Int 57: 487-498, 2000.

30. Aleksandrova K, Mozaffarian D and Pischon T: Addressing the perfect storm: Biomarkers in obesity and pathophysiology of cardiometabolic risk. Clin Chem 64: 142-153, 2018.

31. Jung HJ, Seu YB and Lee DG: Candicidal action of resveratrol isolated from grapes on human pathogenic yeast $\mathrm{C}$. albicans. J Microbiol Biotechnol 17: 1324-1329, 2007.

32. Malhotra A, Bath S and Elbarbry F: An organ system approach to explore the antioxidative, anti-inflammatory, and cytoprotective actions of resveratrol. Oxid Med Cell Longev 2015: 803971, 2015.

33. Cucciolla V, Borriello A, Oliva A, Galletti P, Zappia V and Della Ragione F: Resveratrol: From basic science to the clinic. Cell Cycle 6: 2495-2510, 2007.

34. Gambini J, Inglés M, Olaso G, Lopez-Grueso R, Bonet-Costa V, Gimeno-Mallench L, Mas-Bargues C, Abdelaziz KM, Gomez-Cabrera MC, Vina J and Borras C: Properties of resveratrol: In vitro and in vivo studies about metabolism, bioavailability, and biological effects in animal models and humans. Oxid Med Cell Longev 2015: 837042, 2015.

35. Modi S, Yaluri N and Kokkola T: Strigolactone GR24 and pinosylvin attenuate adipogenesis and inflammation of white adipocytes. Biochem Biophys Res Commun 499: 164-169, 2018.

36. Aguirre L, Fernández-Quintela A, Arias N and Portillo MP: Resveratrol: Anti-obesity mechanisms of action. Molecules 19: 18632-18655, 2014.

37. Baur JA and Sinclair DA: Therapeutic potential of resveratrol: The in vivo evidence. Nat Rev Drug Discov 5: 493, 2006.

(i) $(-)$ This work is licensed under a Creative Commons Attribution-NonCommercial-NoDerivatives 4.0 International (CC BY-NC-ND 4.0) License. 